ESSAY ON THE THEORY OF WHO IS GOD, THE MATRIX PARTICLE AND QUANTUM STAMENS: IN THE FORMATION OF EVERYTHING AND THE UNIVERSE

\title{
THEORETICAL ESSAY
}

SANTOS, Irineu de Oliveira ${ }^{1}$

SANTOS, Irineu de Oliveira. Essay on the theory of who is God, the matrix particle and quantum stamens: in the formation of everything and the universe. Revista Científica Multidisciplinar Núcleo do Conhecimento. Year 06, Ed. 09, Vol. 02, pp. 05-22. September 2021. ISSN: 2448-0959, Access Link: https://www.nucleodoconhecimento.com.br/fisica-en/who-is-god, 10.32749/nucleodoconhecimento.com.br/fisica-en/who-is-god

\section{ABSTRACT}

Given that God is nothing absolute, the only eternally present, immutable and neutral element of any equation that can contain everything we can understand as dimensions, particles, waves, matter, energy, life; where without this force the universe and the reverse could never happen, being a single-hand, undeniable pathway, this essay aims to deal with considerations related to the God particle, as well as describe this particle, here baptized as Matrix Particle and Quantum Stamens (PM and EQ), about its behavior in space, which is intrinsic to the understanding of its existence. This theory is based on own observations and bibliographies already published based on studies on the understanding of what is the greater God and the demigods or galactic Gods, including an analogy of how the universes contain the god endorsed and other of lesser power energy, among other studies related to the theme that deal with the most basic particle to make up the universe, both in its matter, as forms of energy and even predicting the existence of antimatter. Thus, for

\footnotetext{
${ }^{1}$ Experimental Physicist. Economist.
}

RC: 98373

Available in: https://www.nucleodoconhecimento.com.br/fisica-en/who-is-god 
better compression, I describe within this larger structure called "God", which is where there are the fundamental particles, composed of six basic elements that we call in this function as "Quantum Stumers", and an energetic particle with its own light that we call "Matrix Particle" because it is mainly the light of the universe, where without this radiant energy there is no motor life, which is what interests us. That said, in a few words, the results revealed that if the concepts of pmeeq's form and performance are widely studied and understood, these data will enable an advance in terms of energy use, fuel production and even material transport.

Keywords: God, Matrix Particle, Gravitational, Quantum Physics, Quantum Particles.

\section{INTRODUCTION}

The present article is a collection of considerations and conclusions based on studies and observations on what God is, string theory and other studies of the same theme that deal with the most basic particles to be made up of the universe and itself, as well as gives passivity of classical and quantum physical space, as a key element. Behaving within God these elements, both in their formation of matter as forms of energy and even predicting the existence of antimatter.

To describe God outside the religious or philosophical context and especially the particle named authorically (for this theory) as Matrix Particle and Quantum Stumins (PM and EQ), it is also necessary to lecture on its behaviors in space time, which is intrinsic to the understanding of its existence. This is an extremely complex theme, since most of the information is obtained in an observational, theoretical and mathematical way, and a wide understanding of the subject and a certain degree of deductivity are required from the existing studies and now used. Once the concepts of PMeEQ's form and performance are widely studied and understood, in our daily lives, these data will enable an advance in terms of generation and use of energies, with some suggestions described later, also forms of fuel energy production, functioning of the human mind, correlation with the functioning of the universe and our body, relationships with the environment and its forms, whether waves or

$\mathrm{RC}: 98373$

Available in: https://www.nucleodoconhecimento.com.br/fisica-en/who-is-god 
frequencies, and also the transport of matter and acceleration of bodies, or control of their acceleration.

The Matrix Particle is the glue and crossroads of the fundamental particles and for us the most important. It exists encastelada in the union of 6 elastic Estames, and when together, they are released from the background walls of the Universe that create energies and dimensional materials distinguishable in our universe. This tangle is released internally from the walls of the ultra universal balloon, by gravitational rotary motion, by geoelectrospatial exuding discharges present in the wall of the background that makes up the universe, and by bulges of pentadimencionais energies and other frequencies, equal to or above these, which act as an ultra grater, extracting and distributing pieces of this quantum tangle, enabling them to emerge: energy, mass, gravity, attraction, resonance, dark matter and dark energy, polarized or not, movement and time, anti-gravity, antimatter.

\subsection{CLASSIFICATION INTRODUCTION AND THEORETICAL REFERENCES}

God: nothing absolute, everything, the uterus, space to shape matter, which allows frequencies, waves and vibrations, cold, absence of light and movement, space, permeability, the conductor.

Philosophically God is the hall where the meeting balls take place between the Étienne Klein ${ }^{[2]}$, the shocks of higgs ${ }^{[3]}$, the space of Beethoven's music notes ${ }^{[4]}$, the place where Max Karl Ludwig Planck ${ }^{[5]}$ created his theory, among others. God is also briefly well described by Helinando Oliveira, Professor of "Univasf", where he is coordinator of the Laboratory of Impedance Spectroscopy and Organic Materials (LEIMO), in his subject "god and science", however, we disagree in part of this presentation (OLIVEIRA, 2019). God resides in the perfection of our interior, with regard to humans as living, because of course he-god is also within us, regenizing our spacing and maintaining our vibration and temperature while every kind of wave

RC: 98373

Available in: https://www.nucleodoconhecimento.com.br/fisica-en/who-is-god 
crosses us without causing harm and without causing molecular decrease in the spacing of our matter. We know what any spatial imbalance between molecules or even between cells causes, this by talking about living things and their perspectives.

\subsection{CLASSIFICATION INTRODUCTION AND THEORETICAL REFERENCES}

The Matrix and Quantic States particles (PMeEQ) are the particles that create and unite all existing matter and energy in our temporal dimension, they are distributed throughout the universe and make up everything that exists. The Matrix Particle is sustained and/or anchored according to the situation by six fundamental, fundamental nationalities/forces, and may have triangular, square or hexagonal shapes, and the PM has the mass and dimension of 5 of these Quantum stakes, where the sixth anthropic estame/force, is variable in its position and time/force, and may belong to particle "A" or particle "B", as well as particle B can share the string with particle "C". This movement of the joker quantum stake is responsible for every movement within the Universe, because its destabilization moves the PMeEQ forming physical materials and energies, of classical and quantum physics, dimensional, multidimensional, pentadimencionais and exadimensional. But it is also, the PMeEQ the barriers and the insulator, for themselves and for other particles formed and formative, of equal measurement or larger, whether classical and even dimensional quantum.

These forms of energy/wave and matter present in the gravitational shell of the macro universe, are a neutral structure as anchored, and remain neutral until exposed to a natural coercive spiral gravitational force of the universe or caused by the approximation of another dimensional universe. It is not uncommon or unusual for $\mathrm{PMeEQ}$ to have the shape of a flower, dandelion, adding more estamens, because in the quantum world the above can be the bottom and the outside can be the inside, and the connections can be together, but separated, at the present time, but at the same time in the future or in the past, it may occur that the Quantum

$\mathrm{RC}: 98373$

Available in: https://www.nucleodoconhecimento.com.br/fisica-en/who-is-god 
Stumers are in skein, acquiring other properties and characteristics unimaginable at this time.

It is even more important when it is diagnosed that these particles are active and memory-capable materials (CHVYKOV et. al. 2021), they are time-timed, transiting in the three time stages of classical physics and multiple dimensional times, and can give pentadimencionais/exadimensional, classic or quantum leaps.

Within classical physics, speaking of particles and space, the bonds dictate our life from forever, speaking by analogy, clouds are only sustained by the dimension of the molecules that maintain the union and distance between them, this is almost free of gravity, although suffering the Casimir effect ${ }^{[6]}$

\section{DEVELOPMENT AND ORIGIN}

To speak of the interactive properties and capacities of God, in the scientific sense seems to have no practical sense in our current degree of development, but it has, and God is basically located and recognized as author/co-author/chief director, since it is clarified to be the place where everything always occurs, more productively.

Not supporting redundancy to the obvious, this theoretical essay will be described from data collected in other studies, observations and other theories, and much by my own insight, focusing on the beginning of connections and movements.

The phenomenon of the liberation and disaggregation of the Matrix Particles and quantum stuns within the quantum tangle occurs mainly from the background wall of the universe by various phenomena from which God is participatory and there begins the saga, in the sum of the PMeEQ with other PM, and the release of the 6 Gravitational Quantum Estames, where "maybe" are the: tau, electron, múon, graph, $\mathrm{X}$, and "C" (joker), or more likely something smaller and with other characteristics besides quantum, that our mind and understanding do not reach.

RC: 98373

Available in: https://www.nucleodoconhecimento.com.br/fisica-en/who-is-god 
It should be noted that these Quantico Stits are not the strings of Theodor Kaluza, nor of Kaluza Klein ${ }^{[7]}$, thus imputed to them another meaning in the meaning of mooring and distancing of mass in their sum, of polarity, of shapeers of fluid gravity, of lubricants and mainly of movement.

The principle of aggregations and disaggregations happens from the polarized rotational gravitational spiral compression, when one PMeEQ impregnates the other and coexistence breaks up and releases the PM from the restrictive geometry of the Quantum Stumps, leaving free the particles and the stums, these are acquiring other characteristics and properties and also influenced by gravitational singularity and under certain speed conditions, time, space, duration and excitement acquire polarity and can transform into energy, wave matter and time, even all in the same space/time.

When and where singularities begin to happen is in the separation and reorganizations of PM, of the quantic Sustaining Estames. Pms can become materials or energies, and Quantum Stumers become dark matter or dark energy (See attached drawing). The primordial quantum tangle that forms the background walls of the macro Universe, happened at time immemorial by the union and friction of multidimensional energies of several ultra universal balloons, carriers of the billions of galaxies, the clusters, which stopping the movement of friction," solidified"this rotational energy, which called the tangled matrix of the PM and their Quantico Semdes.

The recognizable scale model we know, and more like what we have to compare with energy or neutrality as PM presents, is static electricity. Using analogy again, a good example is a wool mop passed over a car and getting electrostatically charged while receiving the load of matter that was at rest there.

RC: 98373

Available in: https://www.nucleodoconhecimento.com.br/fisica-en/who-is-god 


\subsection{RELEASE OF PARTICLES}

The PMeEQ swaded throughout the universe, from the release of matter from the background walls of the Universe (Theory of conscious creations). The Universe is shaped like a bubble in gums not exactly round, the most real possible analogy is that the Macro universe composed of millions of cluster's is similar to a more rounded stomach, with more walls than a common human stomach, but with input and output exactly as in humans, only that with more onion-like layers, where invisible dimensions float or more precisely at other frequencies outside of our readings by current or observational equipment by simply looking, floating in an incomprehensible existence to our contemporary limited consciousness, within these dimensions I understand that forces that are unknown to us, the where on the material plane, let us say palpable, the walls are detached energetic material when the balloon (stomach) rotates, because there are imperfections, impacts, bumps, gravitational leaps and attraction of the polarized axis of the universe, from which the material that fills space is destitution. The tangles are released from the walls of the macro universe, mainly by the irregular circular rotational motion, and reach the center of the universe, already as galaxies, where circulates a river of horizontal energy from pole to pole, formed by the Matrix Particles and the Quantum Stumens already energized in motion, where new charges create, with the beginning of compression of space gases, the suns and polarize them. These dancing polarizations create planets from the residue of gases, which the suns have not used, and the striated shape of almost all galaxies, and spread PM that lighten the Universe and EQ that seem to create a dark but light-permeable curtain by the little vibration present there.

A two-dimensional example would be a swirl of water inside an ovoid balloon, where they float very thin, multi-layered nets and where each junction or node of this network would be a galaxy of PM and the Quantum Stumps.

Part of the PMeEQ are transformed and created matter, and their presence in certain lapses and occasions of time obeying cosmic influence, reverse the rotation and

RC: 98373

Available in: https://www.nucleodoconhecimento.com.br/fisica-en/who-is-god 
polarity as the movement of a shoal of frightened sardines, or by another analogy, another example, would be a human stomach or a pig crossed by an electric wire, thick, bare, what time is pulling time is transmitting energy. These inversions occur instantly in cosmic time managed by the entireouter walls of the universe, or of galaxies where they are independent of how many trillions of light years it imports.

This happens in our time count, which can be zero, "the" time, so that these inversions and displacements happen, although in the matter affected may take a larger time space to happen, by our perception of what is too much or too little time, or to be a sectoral phenomenon, cosmologically speaking.

The Matrix particle is the fundamental intangible element, which when triggered acts as a reactor or something impregnated with static electricity, can be hard or fluid gravity, can be wave, acting with the Stouts, or even antimatter, particle that surrounds and accompanies all other minor particles existing. These particles, when excited at a certain gravitational force, velocity and time space, can form an immaterial energy tail, like a quantum comet, regardless of the amount of matter that accompanies it, serves as material truth for all particles, even though it also exists within this same particle, composing matter. PMs do not attract in the primordial stage, either by limiting the anchorage of EQs, or because they have no preference or flavor, although they are always together and can act as a relé, but can acquire polarization, and allow the existence of energy with their movements, they have the memory of meaning and movement still in creative immateriality, and transmits this movement when they acquire mass and create energy.

Every measurable space, in the smallest quantically existent dimension, is the Matrix Particle with its Quantum Stumbes. The space of galaxies is matter and antimatter, when charged with energy from quantum particles. The Matrix Particle and the Quantum Stakes, releases, attracts and creates all existing, or missing energy in the universe, is what scholars determine, dark matter and energy and all existing matter, it coexists between two polarities, various dimensions and gravitys, because it is

RC: 98373

Available in: https://www.nucleodoconhecimento.com.br/fisica-en/who-is-god 
neutral until it reaches a certain inertia of speed, space and time. It is static until some force of rotation to excite, the very rotational motion of galaxies exists by influence of the formation that aggregated it, and that maintains the movement of galaxies, suns, planets and stars of any magnitude in flux and reflux.

The ultra universe of ovoid format is crossed by a gravitational axis of classical energetic matter, but also of dimensional, penta or hexa-dimensional forces, rotational in the form of several intertwined DNA spirals. This axis is a material sample of the links between relativistic and quantum theories, present in solid celestial bodies (Ether theory as an element).

Without the Matrix particle and its Quantum Stumens being moved under certain conditions, the background walls would also have stop expelling particles and the walls' own rotation would slow down, collapsing the system. In all situations the Matrix Particle and its Quantum States have to be part of the calculation, such as the tail and mantle in front of an accelerated proton in the HCL, for example, as well as when the static inercial loads multiplied at the end of the route are divided. Always this particle, of the dimension of a "how much" or smaller, is flowing as ultra quantum and gravitational liquid, in order to interact with the physical phenomena of matter, and with energies of various dimensions and frequencies.

The PM theory, in some respects, agrees with Kaluza-Klein's theory, as to the large number of dimensions and as to being Quantum States closed at their ends in most cases. They also agree about the Matrix Particle being found in any of its infinite modes of vibration or neutrality, and may belong to any species, existence, frequency, color or flavor that even composes the human being when one sees the biophotons, which we call and photograph as aura.

\section{CONCLUSION}

Why identify God and name the other elements as Matrix particle and Quantic Stummies (PMeEQ). First, because we have to explain the existence of the crossing

$\mathrm{RC}: 98373$

Available in: https://www.nucleodoconhecimento.com.br/fisica-en/who-is-god 
of galaxies, large negative and positive centers of gravity, which take and repons matter in our ultra dimensional cosmos, and why we call it black holes, as well as the functioning of the magical quantum spacing and its gravitational forms that act in our own existence and as it is everywhere (including within us, where is energy is known as free will and space fluctuations). This truce of shocks between protons, neutrons around the molecules in trillions of combinations needs space, because this support structure of interactions is life, but it is neutral, like our acts and movements, where we determine and determine everything, the future and the paths.

Everything emana from the existence of this primordial force God, and of these PM and $E Q$, they are matter, frequency, dimension, is the meaning of movements and the aggregating gravity, the truss and the glue of the tangle, it maintains balance between attraction and distancing, enabling harmonious existence, and forms of energy, and communication between all existing things, including multidimensional, variable and intelligent frequencies.

In many respects, we agree with the Theory of Functional Convenience of Júnior (2019) and Rovelli (2017), where it is deduced that we create reality from the creation of thought of what is feasible to be reality, which then give rise to reality. The anomaly of water in changing as we observe, touch and handle it, is the greatest proof of our participation in the whole of the universe and of our interference in everything by simple observations and thoughts. If we alter this material, this stellar element $\mathrm{H} 2 \mathrm{O}$, by the simple act of taking it in the hands and taking it to the mouth, interfering in all our body structure, physical and chemical, what can our mind achieve? Can we shape future reality by thought in the aggregation of these matrix particles? The future is already projected by the thought of yesterday, and what we will find in the sinks of space is what our imagination has created, or is it what we have subconsciously known in the formation of fundamental creative energies?

And why this study in the practical, immediate functional aspect? Because in order to break with gravitational ties, develop inexhaustible sources of energy, put a sense

RC: 98373

Available in: https://www.nucleodoconhecimento.com.br/fisica-en/who-is-god 
into the consumption of planetary wealth, especially at this moment of development, and justify our existence, along with the advancement of communications and new centers of management and control, we must pay attention not to be eliminated by the "Al" artificial/mechanical intelligence (OLIVEIRA, 2019). The last mathematical data of study of the adopted path, point to the uncontrol of our creation, in view of the alarming data of destruction that we implement every new day, we must prove that we are descendants of a universal chain of events and that we recognize these beings from God and matters of the study of Theory as superior to us. We must respect them, for by them our permissionaries, we are forgiven. He "God" is something to which even governments submit and attribute value and respect spontaneously, even by adopting in their coins and symbols his name, "GOD", making it clear (for example) that only he can judge.

By the last experiments with "Al", without giving existence to this divinity, we are making room for being judged by logic that can not recognize our mistakes without eliminating us by what we do and practice. We have to know what makes up our limitations, where our barriers are, and seek new paths and other logic plans. Without a logical plan of restriction for "Al", in addition to the 4 laws of Issac Asimov, which may seem illogical for "Al", in the face of the destruction of the world, we are unprotected, so we must predict that all freedom that we are transferring machines today, have limitations in their own failure to not be able to relate to this force greater "God", for instance.

And who is God outside the theological dogmas? Who can God be coldly studying known frequencies and projecting them as a creative force for the formation and aggregation of matter? What can be the interest of something as powerful in tiny beings as we for example? What may be the reason of our existence for such a creature, or such creatures? Ionic energy, which seems little even that of billions of human beings, and even to a lesser extent of other living beings, and perhaps gravity itself, are a universal plan of maintaining grounding to release biocellular energy, are

RC: 98373

Available in: https://www.nucleodoconhecimento.com.br/fisica-en/who-is-god 
possibilities not excluding to justify our dimension, since we have already proved to exist others with other laws of physics acting.

Then we can deduce that there is a higher God (let's assume that he is a complete human being, and that he has a brain that controls everything) and within him several universes each with his own central neurons to command and communicate with the father God. In the center of it, the stomach, which inserts in the father all the energies of the most varied formats, transforms them and redirects them to other dimensions. Within this vast complex, there is an element that is our planet. Seen as energy, we are not matter for this being, but perhaps we are matter for a group of closest energetic neurons, which would be our personal Gods, those who look at us as food, who interfere in our progression of development by voracity, beings living in unimaginable time spaces, with timeless breeding plans, like some Gods for each cluster, that is, for each layer of the stomach, or for each dimension. All wanting to be more powerful or representative neurons before the body of the FATHER/GOD, and some depending on our bio-ionic floating energies, which are time positive, negative time. When they are good, they feed positive beings, and if negative, dark beings. Fact is, we're all the fruits of transformations, of energy in narrow dimensional motion. Our existence itself is an act of violence, we are consumers of matter, it hurts the universe, because we cause imbalance in our own existence. Thoughts and vibration are negative factors, although part of a larger plan of these timeless intelligences, for whom our existential questions are irrelevant and unlogical, since we are inside the system and exist with a purpose.

The smaller Gods, which are not used as a material reference in this Theory, are the indicators and markers that interfere in the most primitive forms of bio-active energy liberators (we), suck and feed the entire chain. It also seems that as the time and evolution of these species of superbeings evolves and moves to another plane or dimension, the beings here also change and create new images of worship, as well as new temples of worship, directing and modulating another type of energy liberation.

RC: 98373

Available in: https://www.nucleodoconhecimento.com.br/fisica-en/who-is-god 
In addition to the human logistical issues of integration with "Al", we must think of new and unprecedented means of acting and exploring our planet, about new means of locomotion, creation of physical spaces lands now inaccessible or expensive if mounted by tangible material physical barriers, such as the seabed and the sky, (space between soil and stratosphere) for example, that does not exist by our degree of development and the possibility of creating forces such as walls of repulsive energies gravity without having to act materially by spending other energies.

This theory can put a different way, knowing the constitution of primordial matter, to hide from gravitational attraction parts of matter that we do not want to suffer external influences, finding the right configuration of the PMeEQ that is creating the attraction, and I leave to the public domain that we can create a darkening bubble around the object we want, even using photons, for gravity not to feel it, such as the walls of a Zeppelin around the object, so that in its fluid form the PMeEQ so that they cannot soak and keep soaked the object of interest of the release of weight and or mass recognition, or energy and frequency that reflects the attraction of $P M e E Q$ in its tiniest forms where it is impregnated, so gravity finds nothing to tie or attract. We must work to waterproof the objects of this fluid energy which is gravity.

This singularity of hiding from the primordial forces, things, people or areas of matter are also the object of this study, the way of traveling in gravity without the disorders of it arising advance in gravity as a scalpel in a gelatin opening and closing without a trace is the goal, along with the brake of respect for a larger entity for "Al".

It is assumed that within some frequency, dimension or wavelength of electrostatic or continuous light, found fluid in quantum engineering, at its various wavelengths, or similar at even higher speeds, is the key to overcoming attraction, and reorienting this force advantageously.

New technologies, including "Al" need a fixed starting point that gives them security, just as we humans also feel this need as having a sky and a solid object to rely on and that is where God comes from, giving us this solidity, because for there to be

RC: 98373

Available in: https://www.nucleodoconhecimento.com.br/fisica-en/who-is-god 
flexibility or fluidity space is needed. Only space in all its forms and dimensions, avoids overheating, which is destructive although creative, in all dimensions, especially in the third and fourth, which is where we live and create our machines. Without spacing, time and space, nothing remains if our new electric cars do not have cooling in the batteries to avoid too much friction, do not serve, as well as quantum/organic computers or not. These refrigerations require space, this has to be taken to the functional side in the integrality of the thought of the new thinking machines, and this space has to be understood as the anchor that maintains and allows the functionality of interactions. Our God and our discernment make up the space and by it is initially composed, and it has to be instilled in calculations as something alive.

God is the womb, the central axis of the universe is the umbilical cord, the matrix particle the egg, the stains the sperm, and the whole is our interpretation of how we see life and creation.

In accordance with the above, Dr. Prof. Elcio Abdalla (2004) reaffirms the theory presented in this work, because it contains a face of the prism and a view on multiple dimensions, bringing relevant aspects, but with another reading, interpretation and dimensional, physical and philosophical results.

Finally, we have reached the point where science and philosophy enter atavistic concerns of the human being, which are no longer practical, technical and useful postulated by physics and effected by technology, and have become increasingly technical and speculative.

At first, it can be said that the origin and structure of the geometry of space time are still unknown. By current science, quantum geometries have quantum operators representing space and its interpretation, previously considered as simple, has become more complex. Moreover, within quantum gravitation, in black holes and at very high temperatures, it is essential to take into account all particles and interactions generated infinitely, according to string theories. Basically, they can still

$\mathrm{RC}: 98373$

Available in: https://www.nucleodoconhecimento.com.br/fisica-en/who-is-god 
influence the extra dimensions of string theories and other $M$ theories, raising the complexity of the problem even further. Some believe that the extra dimensions are already close to the observations. In view of this, general quantized field theories began to deal with these dimensions, and the old idea of Kaluza and Klein, introduced in the 1920s, became part of an almost everyday idea, where other dimensions became ubiquitous. (ABDALLA, 2004).

We are now in a much more speculative zone, where the object of study is no longer related only to research and begins to transcend the same, not only because it is something much larger, as well as our universe, but because it contains the researcher, so that the latter is unable to observe its object of study, since there is no causal relationship between one universe and another. This is the quantum model seen under a new dimension, in which the measure, fundamental to the very interpretation of the theory, is impossible to be realized. It exposes new theoretical constructions based on string theory, with new spatial dimensions, with a transcendent time and, also, with multiple creations of universes (ABDALLA, 2004).

Already what professor Dr. João E. Steiner (2006) and other physicists and scholars put, gives the differentiation of this way of internalizing the universe and its formation. Although they are precisely putting what is discussed today and which, in particular, has the same weight as the flat earth and heliocentric or galactocentric, these observations, studies and results strongly disagree and clash against other studies, including against the Big Bang theory, since for this phenomenon to have actually occurred, the megauniverse could not have a central axis of energy.

Throughout history several cosmological models have been briefly presented. Thus, the evolution of ideas can be understood by the succession of models, such as the theory of the flat Earth, the geocentric model, the heliocentric and the galactocentric. (STEINER, 2006)

Over the past hundred years, scientists have developed a theory that is capable of describing the most complex observations currently taken, and that shows the origin

RC: 98373

Available in: https://www.nucleodoconhecimento.com.br/fisica-en/who-is-god 
of the universe under a model that can be researched scientifically, this being the big bang theory. Recently, this theory has been improved to a new concept, namely that of the inflationary Big Bang. Discoveries made at the turn of the millennium revealed that all knowledge we have today is just the tip of the iceberg present in a universe taken by energy and dark matter whose natures are still unknown (STEINER, 2006).

Putting the above views of how physical laws supposedly through the view of men think and behave, in the face of the inexplicable and the mathematical/physical/religious universe, it is expected that new paths to approaches without moral, realistic reserves will emerge.

\section{PROPOSAL}

The path and direction I think is now open, we will have intelligence and strength of thought with attitudes. I think that this, is the bridge that was missing crossing/ crossing, to unify theories, overcome barriers and break down the empty beliefs of differences, it is all a science only, religion is materiality, as well as the reverse is also true. The latest studies indicate that thought shapes our reality (Jaime Marrone $\mathrm{Jr} /$ Carlo Rovelli) and from reality we create the future.

With the knowledge that God is matter and an integral part of our daily lives, studies and experiments, I think it will be possible to have the gravitational force of a ITAIPU in the space of a pencil tip, make material energy ships of variable shape and accelective movements that go beyond the time limit, create new inexhaustible energy sources such as those available in black holes, where " $\mathrm{N}$ " numbers of Matrix Particles and Quantum Estamens exist, there are new forms of multidimensional materials and energies.

Within the theory, the proposals can be almost infinite, but we always focus on two main proposals for the urgency of the moment that the planet passes, and where we can collaborate, being: 1st encapsulation of objects or things in networks of darkening / concealment of gravity, and 2nd climate control by the attraction or

RC: 98373

Available in: https://www.nucleodoconhecimento.com.br/fisica-en/who-is-god 
repulsion of the suspended aerial oceans, two realities that can be implemented today, with little risk (man's greed).

\section{REFERENCES}

ABDALLA, E. A estrutura do universo, a mecânica quântica e a cosmologia moderna. Revista USP - São Paulo, n. 62, p. 6-29, junho/agosto 2004.

CHVYKOV, P. et. al. Low rattling: A predictive principle for self-organization in active collectives. Revista: Science Vol.: 371, Issue 6524, pp. 90-95. 2021.

JÚNIOR, J. M. Teoria da Conveniência Funcional: Uma reflexão sobre as propriedades fundamentais da matéria. Revista Científica Multidisciplinar Núcleo do Conhecimento. Ano 04, Ed. 07, Vol. 12, pp. 133-157. Julho de 2019. ISSN: 24480959

ROVELLI, C. A realidade não é o que parece: A estrutura elementar das coisas. 1 Ed, Rio de Janeiro: Objetiva, 2017.

OLIVEIRA, H. Deus e a Ciência. Ciência nordestina, 2019.

SANTOS, I. de O. Teoria do Éter como elemento. 12/10/2003 Arquivo Nacional, não publicado, reg. 276.887. Livro 499. Folha 47.

SANTOS, I. de O. Teorias da criação conscientes. 30/07/1998 Arquivo Nacional, não publicado, reg. 156.719. Livro 258. Folha 343.

STEINER, J. E. A origem do universo. Estud. av. vol. 20 n. 58. São Paulo, 2006. Disponível em: https://doi.org/10.1590/S0103-40142006000300022

RC: 98373

Available in: https://www.nucleodoconhecimento.com.br/fisica-en/who-is-god 


\section{ATTACHMENT}

Merely illustrative authorial drawing, where God is the void, and the points are the PM, the rods being the Quantum Stamens, but the drawing could also have been triangular or hexagonal.

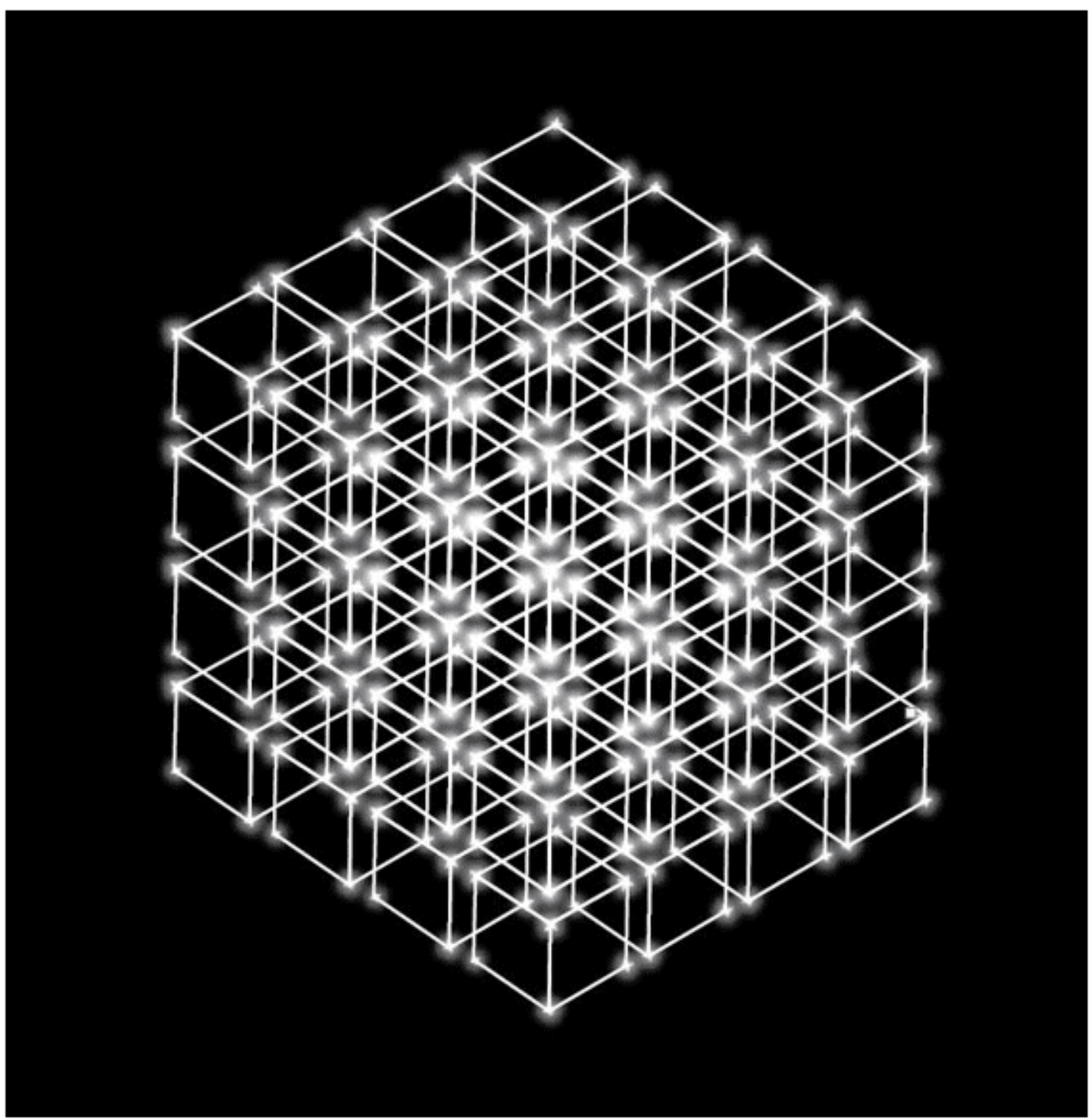

Optical representation of God, Matrix Particles and Quantum Stamens. With PM at rest at the corners of the EQ, showing distance, permeability and neutrality prior to 
gravitational excitation, when PM with its own energy and radiant vibration added together will form mass and energy, and the Stamens will form mass, dark matter and dark energy.

\section{APPENDIX - REFERENCE FOOTNOTE}

2. Étienne Klein, physicist/philosopher Ph.D. École Centrale University, born 01/04/1958. In his book the time that passes, the physicist argues that the future already exists, but we can shape it.

3. Peter Ware Higgs, born 29/05/1929, theoretical physicist, Edinburgh University, Nobel Prize in physics, by the - Particle of God - although he did not give this name, was its editor, also known as "essential particle", the HIGGS boson is considered theoretically as the matter of the universe.

4. Ludwig Van Beethoven, genius, German composer of the transcription period between classical and romanticism birth: 12/1770 Bonn, Germany death:26/03/1827 Vienna, Austria.

5. Max Karl Ludwig Planck, German physicist, Nobel Prize in Physics 1918. Quantum theory, quantum physics, Planck's law.

6. Hendrik Brugt Gerhard, Dutch physicist, (15/07/1909 to 04/05/2000) highlighted by the research "Casimir Effect", LEIDEN University - Two superconductor fluids.

7. Theodor F.E. Kaluza German/Polish mathematical (1885/1945), in 1919 theorizes quantum gravity in loop, that in addition to the 3 dimensions there is a fourth , in a very small circle, and in 1926, Oscar Klein mathematician (1894/1977) proposed the 5th dimension that could be extended or rolled, folded on itself, a folded sheet jumping from one point to another.

Submitted: February, 2021.

RC: 98373

Available in: https://www.nucleodoconhecimento.com.br/fisica-en/who-is-god 
Approved: September, 2021.

RC: 98373

Available in: https://www.nucleodoconhecimento.com.br/fisica-en/who-is-god 